\title{
Editorial
}

\section{Genetics of dilated cardiomyopathy: a molecular maze?}

Dilated cardiomyopathy (DCM) is a heart muscle disease characterised by ventricular dilatation and impaired systolic function, and is a major cause of severe heart failure. Although familial occurrence of DCM has been reported occasionally, the frequency of familial aetiology has been underestimated in the past. Systematic examination of relatives show that probably $>25-30 \%$ of DCM cases are familial. ${ }^{1}$ In this context, it is relevant to discuss whether the rapid development of molecular genetics has allowed us to unravel the molecular defect(s) involved in the disease and led to major breakthroughs in the medical management of the disease.

\section{Familial DCM}

Various modes of inheritance have been reported including $\mathrm{X}$-linked, autosomal recessive, and mitochondrial transmission, but the autosomal dominant forms are the most common. ${ }^{2}$ Although mutations in the cytoskeletal protein dystrophin were identified in 1993 as the cause of X-linked DCM as well as that of Duchenne and Becker type muscular dystrophy, the molecular substrate of inherited forms of DCM remained largely unknown until recently. ${ }^{3}$

To date, scientific evidence suggests that DCM is a highly heterogeneous genetic disease: two X-linked genes have been identified (dystrophin and tafazzin, the latter being responsible for infantile DCM and Barth syndrome) and three other genes have been reported in autosomal dominant DCM ( $\alpha$ cardiac actin, desmin, and lamin $\mathrm{A} / \mathrm{C}$ ). Moreover, seven different chromosomal loci (table 1) have been associated with DCM, and some families are not linked to any of the reported loci, suggesting that other chromosomal loci and genes are involved. ${ }^{4-9}$

The available information provided by the analysis of the publications prompts a number of suggested observations.

Firstly, various phenotypes (that is, clinical features) can be associated with cardiac dilatation and depressed contractile function. This includes muscular abnormalities (dystrophin, desmin, lamin A/C, tafazzin, and 6q23), conduction defects or arrhythmias (lamin A/C, 3p22, 6q23) or the presence of mitral valve prolapse (10q21) or sensorineural hearing loss (6q23-24). Moreover, a similar mutation may result in completely different clinical

Table 1 List of genes and chromosomal loci associated with DCM

\begin{tabular}{llll}
\hline Transmission & Association & Locus & Genes \\
\hline & Pure form & $1 \mathrm{q} 32$ & $?$ \\
& Pure form & $2 \mathrm{q} 31$ & $?$ \\
& Pure form & $2 \mathrm{q} 35$ & Desmin \\
& Pure form & $9 \mathrm{q} 13-22$ & $?$ \\
Autosomal & Pure form & $15 \mathrm{q} 15$ & Cardiac actin \\
Dominant & Conduction disorders & $1 \mathrm{p} 21.2$ & Lamin A/C \\
& Myopathy & $1 \mathrm{p} 21.2$ & Lamin A/C \\
& Conduction disorders & $2 \mathrm{q} 14-22$ & $?$ \\
& Conduction disorders & $3 \mathrm{p} 22$ & $?$ \\
& Conductin disorders + & $6 \mathrm{q} 23$ & $?$ \\
& myopathy & $6 \mathrm{q} 23-24$ & $?$ \\
Autosomal & Mensorineural hearing loss & $10 \mathrm{q} 21-23$ & $?$ \\
recessive & & $?$ & $?$ \\
X-linked & & & \\
& Barth syndrome & Xp21 & Dystrophin \\
& & Xq28 & Tafazzin \\
\hline
\end{tabular}

presentation (both cardiac and non-cardiac) as observed with desmin, ${ }^{5}$ making phenotype-genotype analysis in DCM particularly complex. It is therefore important in the context of a familial DCM to perform a thorough cardiac and non-cardiovascular examination in order to identify abnormalities that might direct genetic analysis toward a specific gene or disease.

Secondly, except for the lamin A/C gene reported in five families, the other genes associated with autosomal dominant DCM have only been reported in a single or in few unrelated families ( $\alpha$ cardiac actin in two families, desmin in one family). This suggests that many of the genetic defects associated with DCM are found in a limited number of families and, so far, there is no evidence of a widespread molecular defect in this disease. Indeed, systematic screening in Japanese, predominantly African, or European DCM populations shows that cardiac actin and desmin genes are rarely responsible for DCM. ${ }^{10}$

Thirdly, the fact that mutations were found in genes coding for cytoskeletal proteins such as dystrophin, desmin, or $\alpha$ cardiac actin has raised the hypothesis that familial DCM is a disease of force transmission whereas hypertrophic cardiomyopathy would be a disease of force generation. However, the exact function of several of the proteins pointed to by molecular analysis, including tafazzin and lamin A/C, a constituent of the nuclear envelope, remains unclear. Therefore, the exact mechanism by which abnormalities of these proteins lead to cardiac dilatation and depressed systolic function is not yet elucidated. Moreover, the fact that mutations in a similar gene, LMNA, may result in apparently unlinked diseases such as Emery Dreyfuss muscular dystrophy, cardiomyopathy or lipodystrophy, ${ }^{11}{ }^{12}$ introduces another degree of complexity.

\section{Modifier genes}

The search for so called disease modifier genes is another fascinating issue. These genes are distinct from the major genes bearing a mutation directly responsible for the disease in an obviously familial context. They may be either directly involved in the pathophysiology of the disease and usually disclosed by case control analyses ("susceptibility genes"), or influence the severity of the disease (morbimortality). Data

Table 2 List of modifier genes associated with DCM

\begin{tabular}{lll}
\hline Genes & Polymorphisms & Survival \\
\hline ACE & I/D & $49 \% v 72 \%$ at 5 years \\
$\beta$ 2 AR & I 1164 Thr & $42 \% v 76 \%$ at 1 year \\
AMPD 1 & Missense mutation & OR 8,6 at 5 years \\
\hline
\end{tabular}

ACE, angiotensin converting enzyme; AMPD 1, adenosine monophosphate deaminase $1 ; \beta 2$ AR, $\beta 2$ adrenoreceptor.

Table 3 List of susceptibility genes associated with DCM

\begin{tabular}{lll}
\hline Genes & Polymorphisms & Odds ratio (95\% CI) \\
\hline PAF acetyl hydrolase & G994T & $1.9(1.3$ to 2.9$)$ \\
SOD 2 & Va116Ala & $2.30(1.27$ to 3.33$)$ \\
HLA-DR & DRB1 1401 & $3.46(1.99$ to 4.93$)$ \\
ETA & Exon 8 C/T & $1.9(1.2$ to 3.0$)$
\end{tabular}

ETA, endothelin type A receptor; PAF acetyl hydrolase, platelet activating factor; SOD 2, super oxide dismutase. 
available so far are scarce (tables 2 and 3). Most studies have focused on the search for an association with an insertion/ deletion angiotensin converting enzyme gene, and conflicting results have been published. In a large case control study, we have ruled out the role of this polymorphism as that of polymorphisms involved in seven other candidate genes as susceptibility factors to DCM. ${ }^{13}$ This points to the importance of the sample size and/or the recruitment conditions of cases and controls. Obviously, there is a need for further studies including functional consequences of the effects of mutations.

Although preliminary, these results suggest that genetic background may influence the development and progression of apparently non-familial DCM. The search for new genetic factors predisposing to the disease is therefore an important issue to be addressed in the coming years.

\section{Clinical perspective}

Owing to the genetic heterogeneity of the disease, and the fact that in many instances only a chromosomal locus is found and not a gene, genetic testing in familial DCM is not yet ready for routine clinical use. Despite the genetic complexity of this disease, the rapid development of the knowledge of the molecular mechanisms of DCM appears promising. This includes early and specific diagnosis of patients at risk, detection of carriers, and early management of subjects at risk in order to prevent the progression of the disease.

Another perspective is the reassessment of clinical classification of DCM based on the phenotypic variability and the molecular abnormalities, taking into account both the cardiac and the non-cardiac symptoms.

Finally, the identification of subgroups of patients based on molecular defects should provide a basis for the devel- opment of cardiovascular pharmacogenomics. If this proves true, molecular genetics of DCM will be a useful tool to find the way out of this clinical dilemma.

Service de Cardiologie,

M KOMAJDA

Hôpital Pitié-Salpétrièr

47-83 Boulevard de l'hôpital,

75652 Paris Cedex 13, France

michel.komajda@psl.ap-hop-paris.fr

1 Michels VV, Moll PP, Miller FA, et al. The frequency of familial dilated cardiomyopathies in a series of patients with idiopathic dilated cardiomyopathy. N Engl F Med 1992;326:77-82.

2 Mestroni L, Rocco C, Gregori D, et al. Familial dilated cardiomyopathy: evidence for genetic and phenotypic heterogeneity. F Am Coll Cardiol 1999;34: 171-90.

3 Muntoni F, Cau M, Ganau A, et al. Deletion of the dystrophin muscle-promoter region associated with $\mathrm{X}$-linked cardiomyopathy. $N$ Engl f Med 1993;329:921-5.

4 Komajda M, Charron P, Tesson F. Genetic aspects of heart failure. Eur $\mathcal{F}$ Heart Failure 1999;1:121-6.

$5 \mathrm{Li} \mathrm{D}$, Tapscoft T, Gonzalez O, et al. Desmin mutation responsible for idio$\mathrm{Li}$, Tapscoft T, Gonzalez O, et al. Desmin mutation responsic dilated cardiomyopathy. Circulation 1999;100:461-4.
pathic dit.

6 Jung M, Poepping I, Perrot A, et al. Investigation of a family with autosomal Jung $M$, Poepping $\mathrm{I}$, Perrot $A$, et al. Investigation of a family with autosomal
dominant dilated cardiomyopathy defines a novel locus on chromosome 2q14-q22. Am f Hum Genet 1999;65:1068-77.

7 Fatkin D, MacRae C, Sasaki T, et al. Missense mutations in the rod domain of the lamin A/C gene as causes of dilated cardiomyopathy and conduction-system disease. N Engl F Med 1999;341:1715-24.

8 Schönberger J, Levy H, Grunig E, et al. Dilated cardiomyopathy and sensorieneural hearing loss. A heritable syndrome that maps to 6q23-24. Circulation 2000;101:1812-18.

9 Brodsky GL, Muntoni F, Mioci S, et al. Lamin A/C gene mutation associated with dilated cardiomyopathy with variable skeletal muscle involvement. Circulation 2000;101:473-6.

10 Tesson F, Sylvius N, Pilotto A, et al. Epidemiology of desmin and cardiac actin gene mutations in a European population of dilated cardiomyopathy. Eur Heart $\mathcal{F}$ In press.

11 Bonne G, Di Barletta MR, Varnous S, et al. Mutations in the gene encoding lamin A/C cause autosomic dominant Emery-Dreyfuss muscular dystrophy. Nat Genet 1999;21:285-8.

12 Shackleton S, Lloyd DJ, Jackson SNJ, et al. LMNA, encoding lamin A/C, is mutated in partial lipodystrophy. Nature Genet 2000;24:153-6.

13 Tiret L, Mallet C, Poirier O, et al. Lack of association between polymorphism of eight candidate genes and idiopathic dilated cardiomyopathy the Cardigene study. F Am Coll Cardiol 2000;35:29-35.

\section{IMAGES IN CARDIOLOGY}

\section{Cardiac hydatid cyst}

Hydatid disease is a parasitic infestation caused by larvae of the echinococcus tapeworm. Cardiac hydatid cyst is uncommon, and we report a case involving a 30 year old woman who had experienced a single syncopal episode. Echocardiography showed a large multivesicle

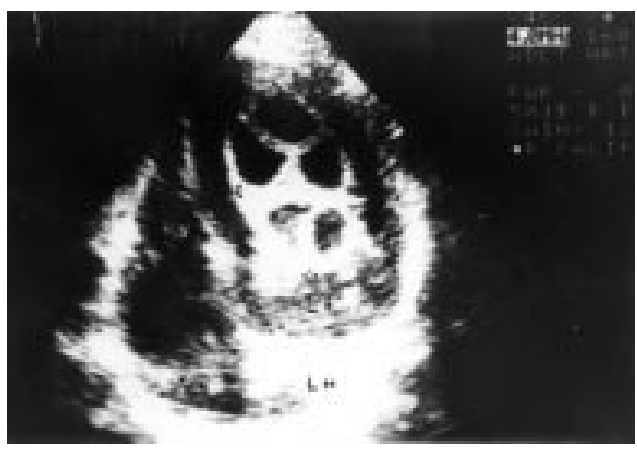

cyst in the ventricular cavity. The cyst, measuring $6 \times 4 \times 5 \mathrm{~cm}$, was localised in the inferoposterior wall of the left ventricle. Thoracic computerised tomography (CT) and magnetic resonance imaging (MRI) also revealed a hypodense mass in the left ventricular cavity. A diagnosis of primary cardiac hydatid cyst was confirmed and total cystectomy was performed.

Echocardiography is the best diagnostic procedure for cardiac hydatid disease, although thoracic MRI and CT, and abdominal $\mathrm{CT}$, are also necessary for detecting the exact location, size and number of disseminated hydatid cysts. 